\title{
Supracondylar Osteotomies of Posttraumatic Distal Humeral Deformities in Young Adults - Technique and Results
}

\author{
Fokko Richard Buß $\beta^{*}, 1$, Arndt-Peter Schulz ${ }^{2}$, Helmut Lill ${ }^{1}$ and Christine Voigt ${ }^{1}$ \\ ${ }^{I}$ Friederikenstift Hospital Hannover, Department of Trauma and Reconstructive Surgery, Humboldtstraße 5, D-30169 \\ Hannover, Germany \\ ${ }^{2}$ University Hospital Schleswig Holstein, Campus Lübeck, Department of Orthopaedics and Trauma, Ratzeburger Allee \\ 160, D-23538 Lübeck, Germany
}

\begin{abstract}
Background: Cubitus varus deformity is the most common late complication after distal humeral fractures in children. Typical symptoms are increasing instability especially the posterolateral rotatory instability (POLRI), lateral elbow pain and cosmetic problems. Different ways of correction have been described but a gold standard has not yet been established.

Methods: In this study the clinical outcome 6,5 months after supracondylar closed wedge osteotomy stabilized with locking plates in four young adults was investigated: three with a posttraumatic varus deformity and one with a posttraumatic valgus deformity of the distal humerus.

Results: All patients showed good or excellent results in the Mayo Elbow Performance Score (MEPS) and the Disabilities of the Arm, Shoulder and Hand score (DASH). In one case, a revision because of a delayed union was necessary, in another case a preexisting pseudarthrosis of the radial epicondyle remained. Neither a residual instability of the elbow joint, nor any significant prominence of the lateral epicondyle was observed.

Conclusion: The supracondylar closed wedge osteotomy stabilized by a locking plate is an effective procedure for the correction of posttraumatic distal humerus deformities in young adults with good final functional results.
\end{abstract}

Keywords: Distal humeral fractures, posttraumatic elbow deformity, supracondylar osteotomy, surgical technique.

\section{INTRODUCTION}

Cubitus varus deformity is the most common late complication after distal humerus fractures in childhood. The incidence of this complication varies from 10 to $50 \%$ [1].

The causes of the deformity are an overgrowth of the lateral condyle or insufficient growth of the medial condyle (e.g. osteonecrosis, growth defect) [2]. Von LAER [3] identifies malrotation as the most important factor in late valgus or varus deformities: due to an interfragmentary rotation with a rotation centre on the fossa olecrani not enough collateral supporting bone remains. This causes a collapse of the medial, less often of the lateral column and leads to a varus or valgus deformity.

Most deformities are diagnosed and corrected in childhood. For a long time, cubitus varus has been regarded as a cosmetic problem but O'Driscoll et al., [4] describes additional problems. Typical complications of the cubitus varus are growing instability: The mechanical axis, the olecranon and the pull-direction of the triceps tendon are shifted medially. This leads to repetitive external rotation torque on the ulna and stresses the lateral collateral ligament

*Address correspondence to this author at the Friederikenstift Hospital Hannover, Department of Trauma and Reconstructive Surgery, Humboldtstraße 5, D-30169 Hannover, Germany; Tel: 0049-511/1292331; Fax: 0049-511/1292405; E-mail: Fokko.Buss@ddh-gruppe.de complex. As a consequence, instability, especially posterolateral rotatory instability (POLRI) occurs [4]. Furthermore, the torn medial triceps tendon may snap over the ulnar epicondyle. In this way or as a consequence of being dislocated anterior to the medial epicondyle -induced by the axis deviation- the ulnar nerve may also be compromised [5]. Pain, instability, snapping triceps tendon and ulnar nerve palsy are the main symptoms of varus deformity of the distal humerus.

The valgus deformity of the elbow joint is much rarer. This deformity occurs mostly as a posttraumatic complication and often as a consequence of a nonunion of a lateral condyle fracture. Symptoms of this deviation can be a growing instability of the medial collateral ligament complex and an irritation of the ulnar nerve induced by stretching.

Previous studies described various corrective osteotomy procedures in children. A high incidence of complications led surgeons to use different techniques without defining a gold standard [6-9]. Three different techniques of supracondylar osteotomies are described: Open/closed wedge osteotomy, dome shaped osteotomy and a three dimensional osteotomy.

We present our surgical technique and the results of four cases: Three young patients with a posttraumatic supracondylar varus deformity and one young patient with a posttraumatic valgus deformity treated by a closed wedge osteotomy. 


\section{MATERIALS AND METHODS}

\section{Patients}

Between 2003 and 2009 four patients with posttraumatic supracondylar humeral deformities were treated with a closed wedge osteotomy. Three varus deformities and one valgus deformity were corrected. Indications for the intervention were pain in the lateral or medial elbow and instability of the lateral or the medial collateral ligament complex. The median age of the patients, one female and three male, was 25,5 (15-29) years. The time of follow up was 6,5 (6-12) months. All deformities resulted as a complication of distal humeral fractures in childhood.

\section{Surgical Technique}

Radiographs of both arms in full extension and supination of the forearm (AP view) were performed prior to the procedure. The axis was set parallel to the humeral diaphysis in relation to the line through the elbow and wrist centre. By reference to the contralateral side the deformity angle and correction angle were determined.

\section{Varus Deformity}

In our institution a closed wedge osteotomy is preferred, because with this technique a good stability can be achieved, so that an early active mobilization of the elbow can be permitted.

The procedure was performed with the patient placed in supine position in all cases, the arm stored on a moveable radiolucent table. For the lateral closed wedge osteotomy we used the radial approach to the distal humerus: After skin incision proximal of the lateral epicondyle, parallel to the humerus and sharp dissection of the subcutis, the fascia between $\mathrm{M}$. triceps brachii and $\mathrm{M}$. brachioradialis was split. The distal humerus could now be prepared. In all steps the anatomic position of the radial nerve had to take into account. In the following step two K-wires were needed: The first one was aligned in a horizontal line parallel to the joint line, directly proximal of the fossa olecrani. The second Kwire was drilled into the bone under fluoroscopy more proximally, meeting the first $\mathrm{K}$-wire on the contralateral cortex in the previously planned angle. A common angle template was used for determining the correct direction (Fig. 1). The bone was cut in the direction of the K-wires using an oscillating saw, strictly parallel to the joint line in the sagittal dimension. The bony wedge could then be removed and the distal fragment shifted slightly medial but not rotated. Under fluoroscopy a locking plate was placed on the lateral edge of the distal humerus as known from stabilizing distal humeral fractures. Finally the fascia and cutis were closed.

\section{Valgus Deformity}

For the correction of a valgus deformity the patient and the arm were positioned in the same way as described above. An ulnar approach was performed, with the skin incision parallel to the distal humerus on the medial epicondyle. The incision was continued through the subcutaneous tissue, the $\mathrm{N}$. cutaneus antebrachii medialis had to be protected. The fascia was cut longitudinally, after which the N. ulnaris had to be located, prepared and secured using a loop. Thereby, the access to the distal humerus was free. The further technique was the same as described above, but from medial.

After the operation, an elbow-immobilizing orthosis was prescribed and only passive motion was allowed for three weeks. For another three weeks, functional aftercare was permitted.

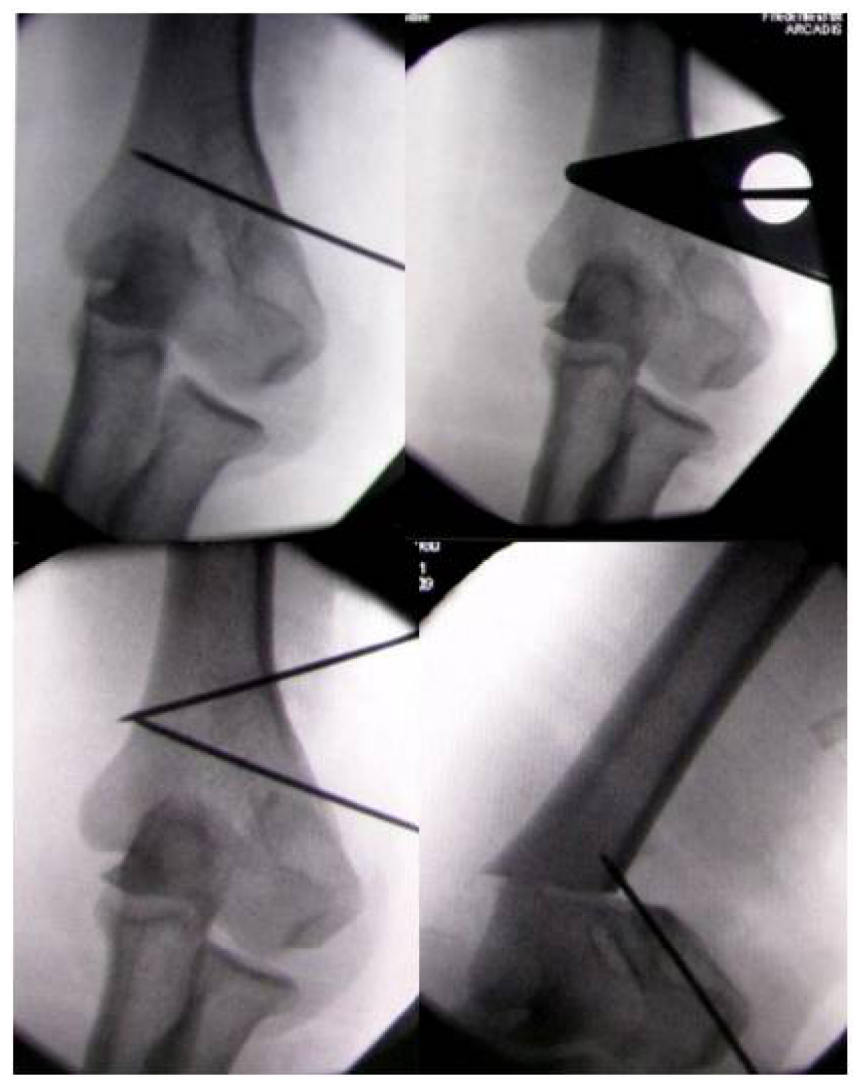

Fig. (1). Intraoperative fluoroscopy: K-wires are set after determining the correct angle with the angle template. After X-ray control the bone was osteotomized.

\section{RESULTS}

\section{Cases}

\section{Patient 1}

Patient one was a 23 year old man who sustained a leftsided supracondylar humerus fracture at the age of nine years, which led to a valgus deformity of the elbow. His symptoms were pain and snapping at the medial side of the elbow. The examination revealed pain on palpation at the medial epicondyle and a significant laxity of the ulnar collateral ligament complex. Radiographs revealed a valgus deformity of $40^{\circ}$. Surgical procedure as described above proceeded without complication. A follow up radiograph at 2,5 months showed the breakage of two screws next to the osteotomy zone. In suspicion of a delayed union due to too much micro-movement and resulting screw breakage, a revision with partial re-osteosynthesis was performed 11 weeks after primary intervention. Seven months after the index procedure the patient was completely asymptomatic and the range of motion (ROM) of the elbow was painless, with extension $0^{\circ}$ and flexion $135^{\circ}$, supination $70^{\circ}$ and 
pronation $80^{\circ}$. The clinical examination revealed no instability of the elbow joint.

\section{Patient 2}

Patient two was a 28 year old man who had a supracondylar humerus fracture on the left side at the age of eight. The fracture was treated conservatively with a cast. His main symptom was persistent pain in the lateral elbow. Pain on the radial compartment could be reproduced by local pressure and varus-stress. In addition, there was a posterolateral rotatory instability. The motion of the elbow was not limited in any way and the neurological examination showed no deficits. The physical and radiological examination revealed a varus deformity of the elbow of $20^{\circ}$ (Figs. 2, 3). The patient underwent lateral closed wedge osteotomy and follow-up treatment in the above-mentioned way. The follow-up examination six months later showed a consolidation of the bone (Fig. 4). The ligaments were stable; the elbow was pain-free and showed a full range of motion with extension $0^{\circ}$ and flexion $140^{\circ}$, supination $80^{\circ}$ and pronation $90^{\circ}$ (Fig. 5).
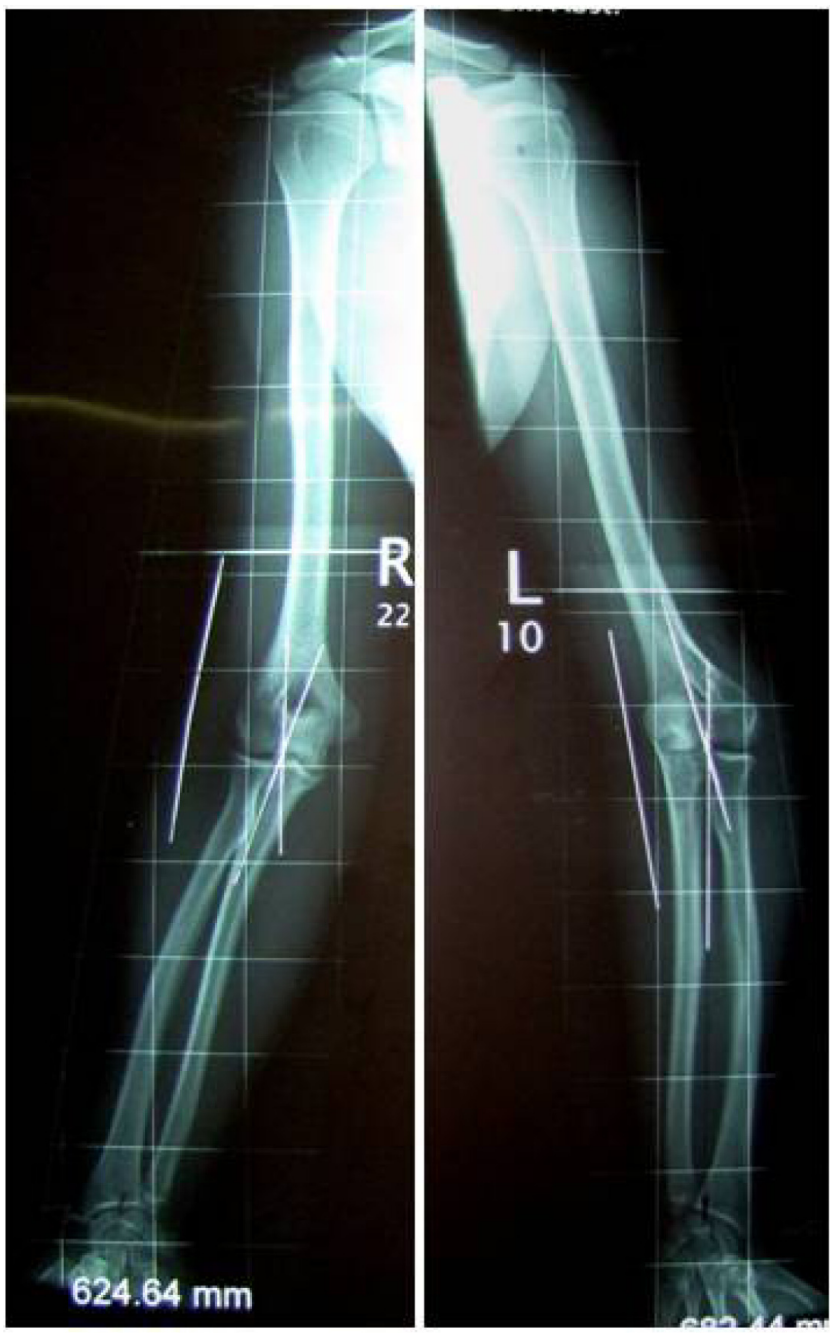

Fig. (2). Patient Two: Preoperative X-rays of both arms with the determined axes: $10^{\circ}$ valgus on the right side and $20^{\circ}$ varus on the left side.

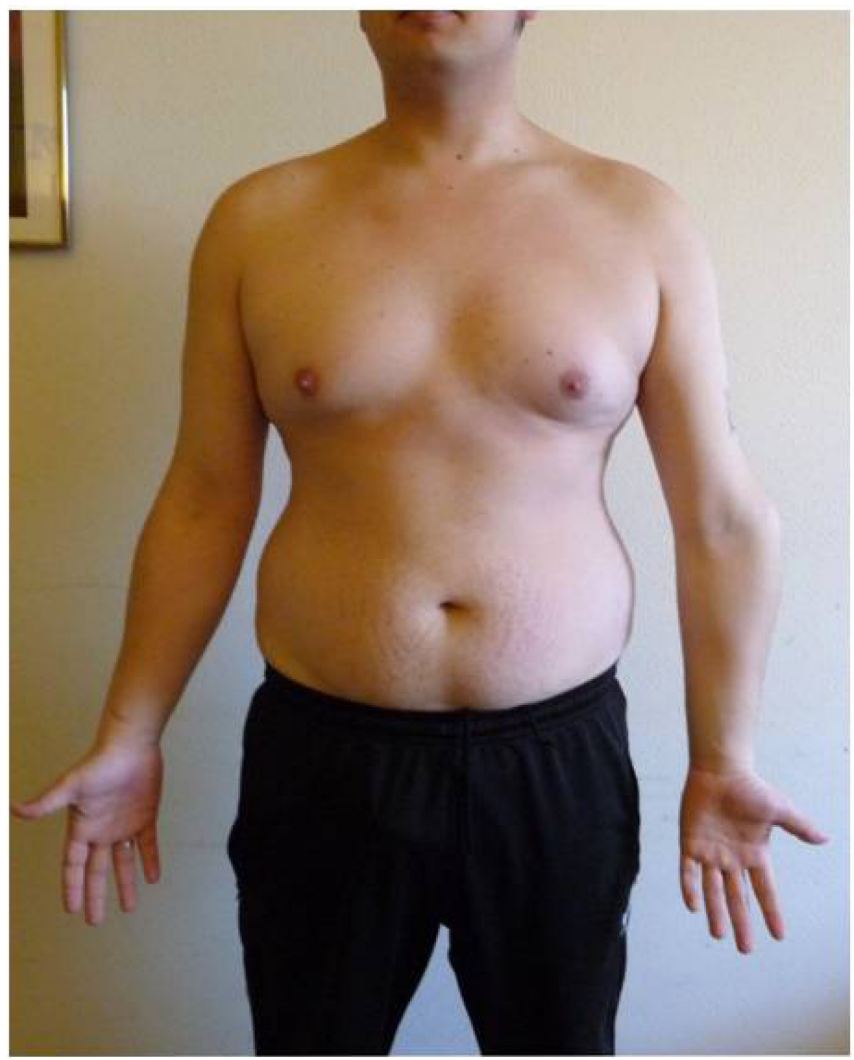

Fig. (3). Clinical preoperative investigation: The $20^{\circ}-$ varus deformity of the right elbow.

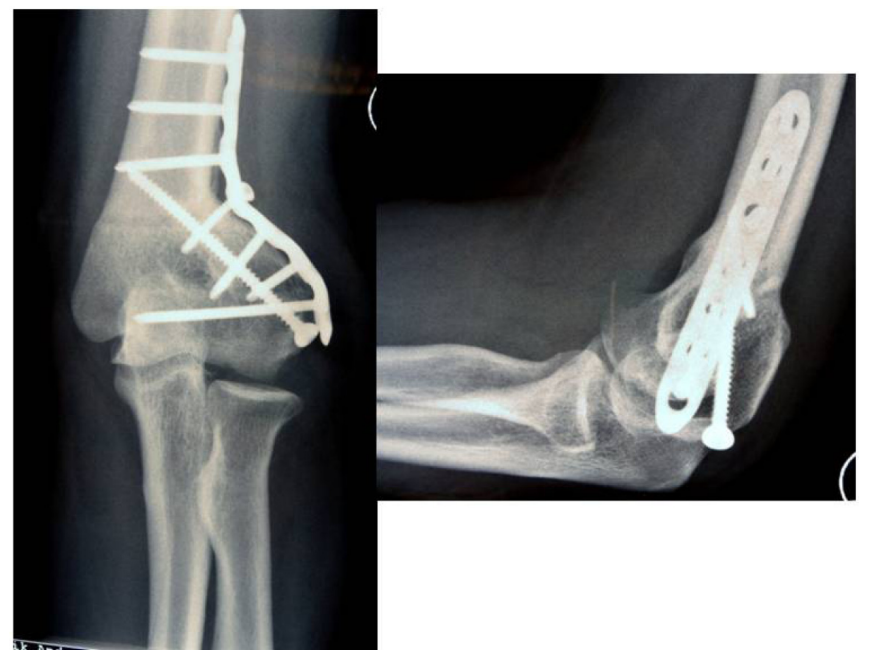

Fig. (4). The X-ray six months after index operation shows a good correction and complete bony consolidation.

\section{Patient 3}

Patient three was the only girl and also the youngest with 15 years of age at surgery. After a conservatively treated right-sided supracondylar humerus fracture at the age of nine, she developed pain in the lateral epicondyle. Her elbow had a full ROM without any sign of instability. The X-ray showed a varus deformity of $15^{\circ}$. After lateral closed wedge osteotomy, a correction to $10^{\circ}$ valgus was achieved. Six months postoperatively the patient was completely 


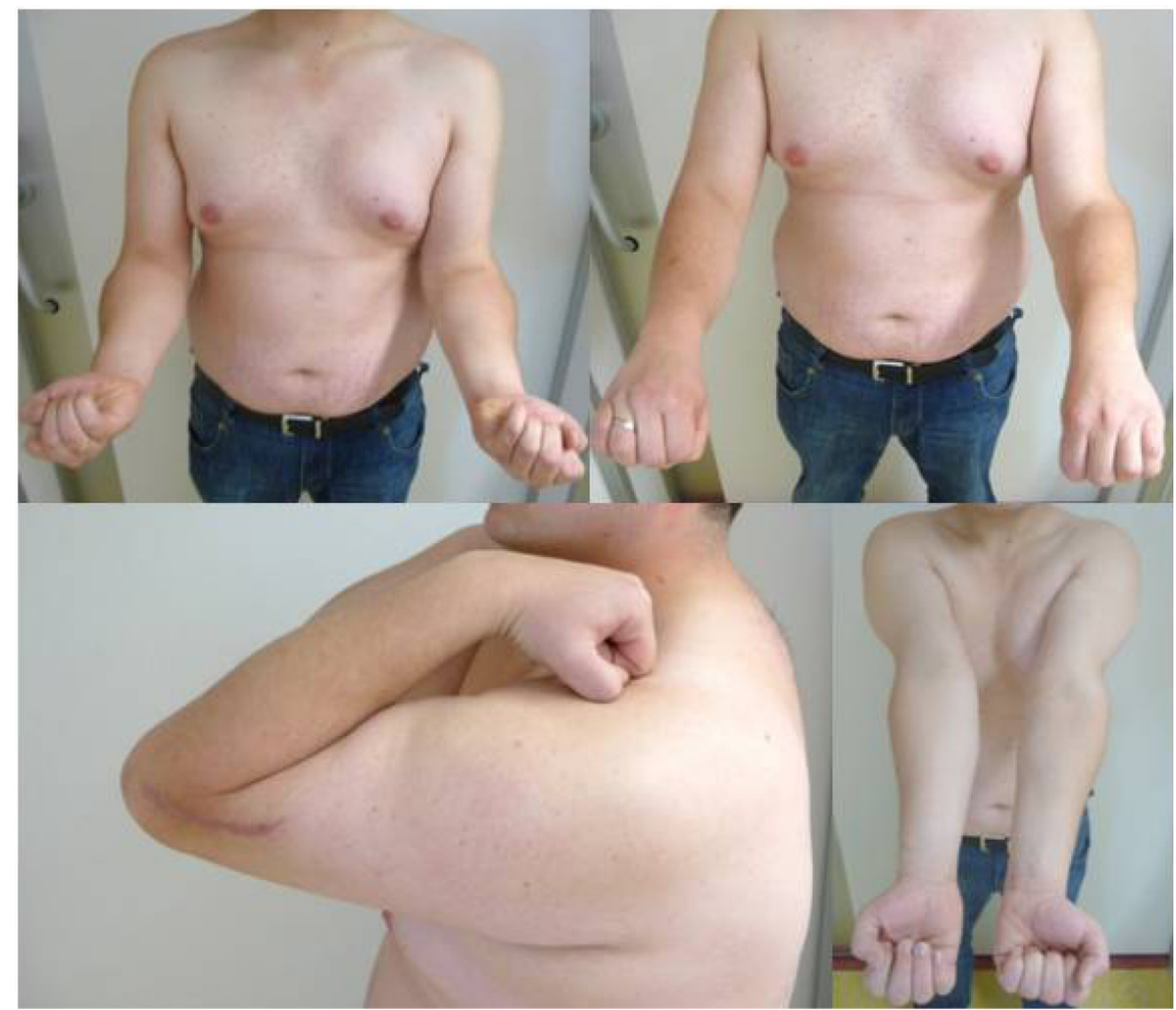

Fig. (5). Functional outcome six months postoperative: Full range of motion.

asymptomatic and the radiographs demonstrated complete consolidation of the osteotomy. The clinical examination showed stable ligaments. The ROM was extension $0^{\circ}$ and flexion $140^{\circ}$, supination $80^{\circ}$ and pronation $85^{\circ}$. No complications had occurred. One year after the index procedure the implants were removed.

\section{Patient 4}

Patient four was male and 29 years old at time of intervention. He had suffered a fracture of the radial condyle at the age of nine. His main symptom was a persistent pain in lateral condyle region. On clinical examination the ROM was extension $0^{\circ}$ and flexion $130^{\circ}$, supination $70^{\circ}$ and pronation $90^{\circ}$. There was a local pain on palpation of the radial condyle and the lateral ligament complex was instable. The X-ray showed a prominent radial epicondyle with a strong pseudarthrosis of the epicondyle and a varus deformity of $15^{\circ}$. After lateral closed wedge osteotomy with a correction to $10^{\circ}$ valgus, revision of the pseudarthrosis and a fixation of the lateral ligament complex the postoperative course were uneventful. On follow up, the above-mentioned symptoms had decreased but had not disappeared completely. The clinical examination showed a good ROM with an extension lag of $10^{\circ}$ and flexion $135^{\circ}$, supination $70^{\circ}$ and pronation $90^{\circ}$. There was no sign of residual instability. However, there was pain on palpation of the lateral epicondyle. The further radiological investigation showed a persistent hypertrophic nonunion of the radial epicondyle. We recommended a revision but the patient has not yet decided whether to undergo another operation.
During follow up six to twelve months after the index operation, all patients underwent radiography of the elbow, which demonstrated complete consolidation of the osteotomy. The anatomical elbow axis was $10^{\circ}$ valgus in all patients. One patient with a delayed union and implant breakage required surgical revision.

The postoperative clinical parameters and score results are shown in Table 1. All patients had a painless range of motion with a median extension of $0^{\circ}\left(0\right.$ to $\left.-10^{\circ}\right)$ and flexion of $137,5^{\circ}\left(130\right.$ to $\left.140^{\circ}\right)$. The median rotation was $75^{\circ}$ ( 70 to $80^{\circ}$ ) for supination and $87,5^{\circ}\left(80\right.$ to $\left.90^{\circ}\right)$ for pronation. No residual instability and no prominence of the lateral epicondyle could be found.

In the follow up examinations patients 1 to 3 were completely asymptomatic and had excellent results in the MEPS [10] and the DASH [11]. In comparison to preoperative measurements the results for the MEPS improved in all four patients (Table 1). Patient 4 is still symptomatic but has good results with $85 / 100$ points for the MEPS and 10,8 for the DASH. In this case, the X-ray and the CT showed a persistent hypertrophic nonunion.

\section{DISCUSSION}

Cubitus varus deformity is a common late complication of supracondylar fractures in the childhood. There are various options for correction of distal humerus deformities. Different ways of osteotomies, for example dome osteotomy, three dimensional osteotomy, step cut osteotomy or wedge osteotomies have been described [12-14]. Different methods 
Table 1. Clinical Outcome and Score Results After Supracondylar Closed Wedge Osteotomies

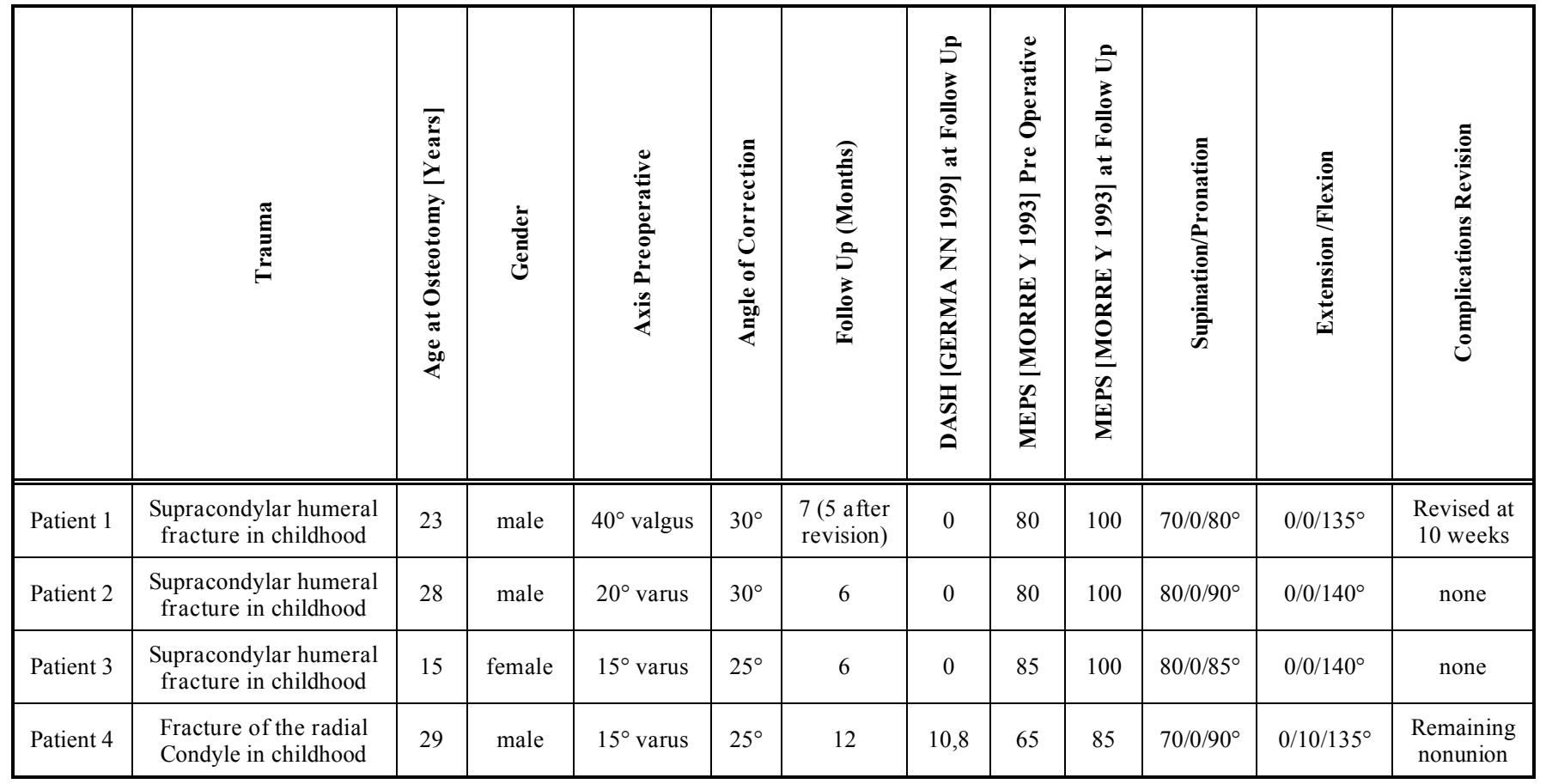

of fixation with $\mathrm{K}$-wire, external fixator, screws or plates have been published $[2,7,15]$.

Most published studies focused on patients in the childhood or adolescence [7, 14-16]. So far, only a few studies have dealt with the correction of supracondylar deformities in adult patients. HAHN et al., [12] investigated the outcome after supracondylar dome osteotomy and KIM et al., [13] found good results for the supracondylar step cut osteotomy in adults. In a previous study of MOON et al., [17] young adults were examined after undergoing lateral closed wedge osteotomy. In contrast to us, he used a nonlocking single plate fixation. GONG et al., [18] performed an oblique closed wedge osteotomy fixed with lateral plating and a lag screw. He treated middle aged patients and revealed good functional results. Our study investigates the clinical and radiographic results after supracondylar humerus closed wedge osteotomies with a locking plate fixation in young adults.

This study shows good clinical results, all patients had good or excellent results in the Mayo elbow performance score [10] and the DASH score [11]. One revision with a reosteosynthesis was performed because of a delayed union. One revision because of a persistent nonunion of the radial epicondyle but not of the osteotomy is pending. Other studies with other osteotomy methods and different fixations showed similar results and complication rates. YUN et al., [14] reported of excellent results in $91 \%$ of cases, in which supracondylar humerus deformity was corrected with reverse- $\mathrm{V}$-osteotomies fixed with $\mathrm{K}$-wires in children. In this study two patients of twenty-two had complications: One had an intra-operative fracture of the step cut spike, the second had temporary ulnar nerve palsy. HAHN et al., [12] reported about 19 cases of correcting supracondylar deformity with a dome osteotomy fixed with plates and screws. Thirteen patients achieved excellent and six good results, no complications were observed. Also TAKAGI et al., [15] found that different methods seem to achieve similar results. He examined patients with different osteotomies: closed wedge-, dome shape-, step cut- and three dimensional osteotomies. K-wires were used for fixation. The 86 treated patients were on average 11,1 years (range, 3 to 31 years) old and were followed up 35 months postoperatively. The study observed differences regarding radiological outcome: The group with multiplanar correction had an average loss of correction of $3,6^{\circ}$, the group with uniplanar correction $0,7^{\circ}$. In this study, complications occurred in six patients.

\section{Lateral Epicondyle Prominence}

In our study no subjective or objective prominence of the lateral epicondyle was observed. This could be due to two factors:

\section{The Surgical Technique}

When the medial cortex is cut as described above, the distal fragment can shift medially. WONG et al., [19] reported about 14 of 22 patients with a significant prominence of the lateral epicondyle. He found that this complication is caused by only hinging the distal fragment on the medial cortex. So he also recommended cutting the cortex and letting the distal fragment shift medially.

\section{The Early Training of the Arm}

Atrophy of the muscles as a consequence of immobilization also leads to lateral epicondyle prominence [20]. With a stable fixation early active mobilization could be permitted and atrophy prevented.

\section{Varus Deformity Accompanied by Nonunion}

One patient in our study is still symptomatic and the radiography shows a persistence of the preexisting nonunion of the radial epicondyle with a consolidated osteotomy. His 
main clinical symptoms resulted from nonunion of the lateral condyle, deformation and instability. TIEN et al., [16] reported about 8 similar cases in which a combined varus deformity and a nonunion of the radial condyle were corrected through a dome osteotomy combined with a screw fixation of the lateral condyle. Patients in this study were children at the average age of 8,6 years. The study observed excellent or good results for six patients and fair results for two patients. In the last two cases symptoms decreased but did not disappear. The combination of varus deformity and nonunion of the radial condyle seems to be special in view of the clinical symptoms and is a challenging combination for the surgeon. Further studies are needed to improve the success rate of the surgical treatment of these special cases.

\section{Necessity for Further Correction?}

Another question is whether to correct other dimensions because of complex deformities. Often the varus deformity is accompanied with an internal rotation or a hyperextension of the humerus. In our survey no further significant deformities which had to be corrected were observed. TAKGI et al., [15] found that it is not necessary to correct an internal rotation because the range of motion and the activities of daily living seem not to be significantly affected by this deformity. Such a rotation defect is difficult to define correctly and is also difficult to correct. A hyperextension deformity should be corrected in adults when it is significant and restricts the range of motion.

\section{Limits of the Study}

The mayor limits of this study are the small number of patients and the heterogeneity of the group (one valgus deformity, three varus deformities, one of which included a pseudarthrosis). Furthermore, the study design is retrospective.

\section{CONCLUSION}

The supracondylar closed wedge osteotomy with a medial fragment shift and a single locking plate fixation as described in this study is a sufficient method to treat posttraumatic deformities in young patients with good functional results. Compared with other methods (dome osteotomy, three dimensional osteotomy, double plating) it is less invasive. The varus deformity combined with a pseudarthrosis of the radial epicondyle seems to be a special case with a higher risk of complications and failure. Further studies are needed to improve the success rate of the surgical treatment of these cases.

\section{ACKNOWLEDGEMENT:}

None declared.

\section{CONFLICT OF INTEREST}

The authors declare that they have no conflict of interest.

\section{REFERENCES}

[1] Laer Lv. In: Laer Lv, Frakturen und Luxationen im Wachstumsalter. Stuttgart, Thieme Verlag. 2007; 118-90.

[2] Voss FR, Kasser JR, Trepman E, Simmons E, Hall JE. Uniplanar Supracondylar humeral osteotomy with preset Kirschner wires for posttraumatic cubits varus. J Pediatr Orthop 1994; 14 (4): 471-8.

[3] Laer Lv. The supracondylar fracture of the humerus in children. Arch Orthop Trauma Surg 1979; 95 (1-2): 123-40.

[4] O'Driscoll SW, Spinner RJ, McKee MD et al. Tardy posterolateral rotatory instability of the elbow due to cubitus varus. J Bone Joint Surg Am 2001; 83-Am (9): 1358-69.

[5] Spinner RJ, O'Driscoll SW, Davids JR, Goldner RD. Cubitus varus associated with dislocation of both the medial portion of the triceps and the ulnar nerve. J Hand Surg Am 1999; 24(4): 718-26.

[6] French PR. Varus deformity of the elbow following supracondylar fractures of the elbow in children. Lancet 1959; ii: 439-41.

[7] Handelsman JE, Weinberg J, Hersch JC. Corrective supracondylar humeral osteotomies using the small AO external fixator. J Pediatr Orthop B 2006; 15: 194-7.

[8] McCoy GF, Piggot J. Supracondylar osteotomy for cubitus varus. The value of the straight arm position. J Bone Joint Surg Br 1988; 70-B: 283-6

[9] Oppenheim WL, Clader TJ, Smith C, Bayer M. Supracondylar humeral osteotomy for traumatic childhood cubitus varus deformity. Clin Orthop Relat Res 1984; 188: 34-9.

[10] Morrey BF, An KN, Chao EYS. In: Morrey BF, The Elbow and Its Disorders. $2^{\text {nd }}$ ed. Philadelphia, W. B. Saunders. 1993; 86-9.

[11] Germann G, Wind G, Harth A. Der DASH-Fragebogen - Ein neues Instrument zur Beurteilung von Behandlungsergebnissen an der oberen Extremität. Handchir Mikrochir Plast Chir 1999; 31: 149-52

[12] Hahn SB, Choi YR, Kang HJ. Corrective Dome osteotomy for cubitus varus and valgus in adults. J Shoulder Elbow Surg 2009; 18: 38-43.

[13] Kim HT, Lee JS, Yoo CI. Management of cubitus varus and valgus. J Bone Joint Surg Am 2005; 87A: 771-80.

[14] Yun YH, Shin SJ, Moon JG. Reverse V osteotomy of the distal Humerus for the correction of cubitus varus. J Bone Joint Surg $\mathrm{Br}$ 2007; 89B: 527-31.

[15] Takagi T, Takayama S, Nakamura T, Horiuchi Y, Toyma Y, Ikegami H. Supracondylar osteotomy of the humerus to correct cubitus varus: Do both internal rotation and extension deformities need to be corrected? J Bone Joint Surg Am 2010; 92: 1619-26.

[16] Tien YC, Chen JC, Fu YC, Chih TT, Hunag PJ, Wang GJ. Supracondylar dome osteotomy for cubitus valgus deformity associated with a lateral condylar nonunion in children. J Bone Joint Surg Am 2005; 87-A: 1456-63.

[17] Moon MS, Kim SS, Kim ST, et al. Lateral closing wedge osteotomy with or without medialisation of the distal fragment for cubitus varus. J Orthop Surg Res 2010; 18(2): 220-3.

[18] Gong HS, Chung MS, Oh JH, Cho HE, Baek GH. Oblique closing wedge osteotomy and lateral plating for cubitus varus in adults. Clin Orthop Relat Res 2008; 466 (4): 899-906.

[19] Wong HK, Lee EH, Balasubramaniam P. The lateral condylar prominence: A complication of supracondylar osteotomy for cubitus varus. J Bone Joint Surg Br 1990; 72: 859-61.

[20] DeRosa GP, Graziano GP. A new osteotomy for cubitus varus. Clin Orthop Relat Res 1988; 236: 160-5. 